23

\title{
Математическая модель распространения лазерного излучения в морской воде
}

\author{
(C) Н.М. Костылёв, В.Я. Колючкин, Р.О. Степанов \\ МГТУ им. Н.Э. Баумана, \\ 105005 Москва, Россия \\ e-mail: kostylevnm@gmail.com
}

Поступила в редакцию 31.05.2019 г.

В окончательной редакции 31.05.2019 г.

Принята к публикации 04.06.2019 г.

На основе известных моделей гидрооптических характеристик слоя морской воды в спектральном диапазоне 530-550 nm предложена математическая модель распространения лазерного излучения. Математическая модель получена путем решения уравнения переноса оптического излучения для узкого пучка с использованием малопараметрической аппроксимации индикатрисы рассеяния слоя морской воды. Модель позволяет определить пространственное распределение облученности на заданном расстоянии от источника лазерного излучения по известной глубине видимости диска Секки. Приведены результаты численных экспериментов для акваторий с известными гидрооптическими характеристиками.

Ключевые слова: подводная система видения, первичные гидрооптические характеристики, перенос оптического излучения, мутная среда.

DOI: $10.21883 /$ OS.2019.10.48357.170-19

\section{Введение}

Разработка систем технического зрения (СТ3), предназначенных для обеспечения функционирования подводных роботизированных аппаратов, является важной научно-технической задачей. Такими системами оснащаются автономные, а также дистанционно-управляемые необитаемые подводные аппараты, которые используются для поиска затонувших объектов, нейтрализации подводных мин, исследования морского дна, укладки или инспекции подводных линий связи и трубопроводов и др. Для выполнения подобных задач наибольшее применение находят СТЗ активного типа с лазерной подсветкой, так как работоспособность таких систем не зависит от условий естественной освещенности подводных объектов. Специфика функционирования СТЗ в подводных условиях обусловлена особенностями распространения оптического излучения в морской воде, которая обладает свойствами рассеивающей среды. Такая среда не только ослабляет мощность оптического излучения, но также вносит линейные искажения изображений регистрируемых объектов.

Чтобы учесть эти факторы при разработке СТЗ для подводных аппаратов, необходима математическая модель распространения излучения в слое морской воды. В общем случае для создания такой модели необходимо иметь достаточно полную информацию о характеристиках воды для заданной акватории. Существуют различные математические модели распространения излучения в слое морской воды. В некоторых моделях $[1,2]$ в качестве исходных данных используют концентрации примесей в воде (пигментов фитопланктона, минераль- ной взвеси и желтого вещества). В других $[3,4]$ исходными данными является совокупность гидрооптических характеристик воды.

В настоящее время в связи со сложностью полноценные измерения гидрооптических характеристик и состава морской воды для широкого круга акваторий не проводятся. Наибольшее количество измерений в различных акваториях по всему миру проведено по наиболее легко измеряемым параметрам: показателю ослабления $\varepsilon$ и глубине видимости диска Секки $Z_{D}$.

Глубина видимости диска Секки $Z_{D}$ измеряется при погружении белого стандартного 30-сантиметрового диска под воду до полного исчезновения его различимости. По сравнению с другими гидрооптическими характеристиками $Z_{D}$ является наиболее легко измеряемой, так как для его измерения требуется только белый диск и размеченный трос. Данные о распределении $Z_{D}$ в водах Мирового океана представлены на многочисленных картах и атласах. В настоящей работе для оценки $Z_{D}$ был использован атлас [5], данные из которого приведены в таблице.

Для разработчиков систем технического зрения наибольший интерес представляют модели распространения излучения, использующие в качестве исходных данных только глубину видимости диска Секки $Z_{D}$. Однако существующие модели, в которых используется только этот параметр, не являются в достаточной степени адекватными, так как в них не учтена зависимость показателя ослабления $\varepsilon$ от $Z_{D}$ для различных акваторий.

Целью исследований, изложенных в настоящей работе, является разработка более универсальной математической модели распространения излучения в морской 
Прозрачность по белому диску в морях и заливах

\begin{tabular}{l|c}
\multicolumn{1}{c|}{ Район } & $Z_{D}, \mathrm{~m}$ \\
\hline Черное море & $5-28$ \\
Феодосийский залив & 14 \\
Балтийское море & $6-12$ \\
Средиземное море & $20-50$ \\
Баренцево море & $6-24$ \\
Карское море & $2-25$ \\
Панамский залив & 10 \\
Бенгальский залив & 45
\end{tabular}

воде, которая основана на использовании видимости диска Секки как единственного показателя этой среды.

\section{Модель гидрооптических характеристик}

Для достижения поставленной цели с учетом минимального количества априорных данных о характеристиках воды в акватории необходимо принять ряд ограничений для разрабатываемой модели. Во-первых, в разрабатываемой модели было принято ограничение спектрального диапазона от 530 до $550 \mathrm{~nm}$. В данном спектральном диапазоне наблюдается минимальное ослабление оптического излучения в водной среде. Соответственно целесообразно выбирать лазерный излучатель для СТ3 с соответствующей рабочей длиной волны. Например, в качестве источника излучения СТ3 может быть использован твердотельный лазер с диодной накачкой на второй гармонике с рабочей длиной волны $527 \mathrm{~nm}$. Во-вторых, следует учесть, что зависимость между параметром $Z_{D}$ и другими гидрооптическими характеристиками определяется типом акватории по классификации Мореля. По данной классификации существует два типа акваторий: Case 1 и Case 2. В водах Case 1 концентрация фитопланктона превышает концентрацию минеральной взвеси, в Case 2 - наоборот. Чаще всего к акватории Case 1 относят воды открытого океана, к Case 2 - прибрежные воды. В-третьих, будем считать, что соотношения между первичными гидрооптическими характеристиками (ПГХ) воды подчиняются модели О.В. Копелевича [6].

Под первичными понимаются такие гидрооптические характеристики, значения которых зависят только от содержащихся в воде веществ и не зависят от условий освещения. Знание ПГХ необходимо и достаточно для описания процесса распространения излучения в водной среде [7]. К ним относятся показатель поглощения $k_{a}$, показатель рассеяния $\sigma$, а также индикатриса рассеяния $x(\gamma)$ водной среды. Показатель $Z_{D}$ не относится к ПГХ, так как зависит от условий естественного освещения при наблюдении диска Секки.

Математическая модель основана на решении уравнения переноса оптического излучения в малоугловом приближении с учетом принятого описания ПГХ. Подоб- ная модель была представлена в [8]. В качестве исходных данных авторами данной работы использовано только значение $Z_{D}$, связь которого с показателем ослабления $\varepsilon$ определялась формулой

$$
\varepsilon=\frac{\alpha}{Z_{D}}
$$

Однако соотношение (1) не является универсальным, так как применительно к различным акваториям значение параметра $\alpha$ варьирует в пределах от 0.8 до 10 . Это свидетельствует о том, что глубина видимости диска Секки не связана однозначным образом с показателем $\varepsilon$ ослабления света. Для примера на рис. 1 приведена зависимость $\varepsilon$ от $Z_{D}$ в прибрежных зонах Черного моря (Case 2) и открытых водах Северного Ледовитого океана (Case 1). Из приведенных графиков следует, что для акваторий разного типа при одинаковой глубине видимости диска Секки показатель ослабления может отличаться более чем в 2 раза.

Анализ экспериментальных данных по измерению показателя ослабления света $\varepsilon$ и глубины $Z_{D}$ видимости диска Секки в различных экспедициях позволил установить, что для акваторий одного типа по классификации Мореля (Case 1 или Case 2) зависимость $\varepsilon$ от $Z_{D}$ достаточно устойчива и описывается формулой

$$
\varepsilon=C_{1} Z_{D}^{-C_{2}},
$$

где $C_{1}$ и $C_{2}-$ константы для одного типа акваторий.

В работе [9] показано, что в узком спектральном диапазоне в окрестности $540 \mathrm{~nm}$ для каждого из типов акваторий (Case 1 или Case 2) зависимость глубины видимости диска Секки от показателя ослабления наиболее устойчивая. На основании данных, представленных в работах [10-13], нами были определены значения коэффициентов $C_{1}$ и $C_{2}$, входящих в уравнение (2) для каждого типа акваторий.

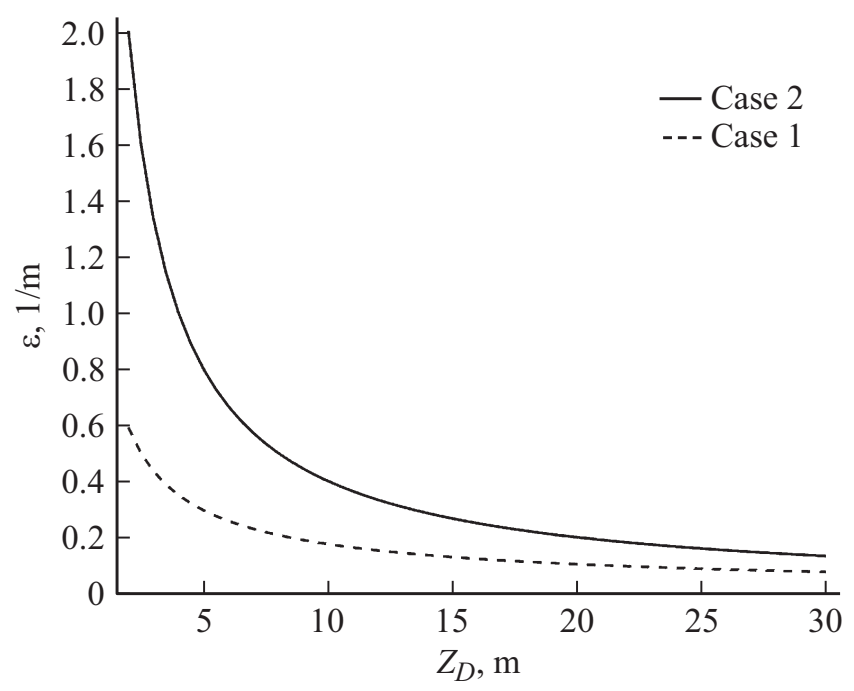

Рис. 1. Зависимость $\varepsilon$ от $Z_{D}$ в прибрежных водах и водах открытого океана. 
Для акваторий типа Case 1

$$
\varepsilon=0.9891 Z_{D}^{-0.741} \text {. }
$$

Для акваторий типа Case 2

$$
\varepsilon=3.0213 Z_{D}^{-0.897} \text {. }
$$

Таким образом, были получены устойчивые для одного типа акваторий и спектрального диапазона в окрестности $540 \mathrm{~nm}$ соотношения (3) и (4) между показателем $\varepsilon$ ослабления и глубины $Z_{D}$ видимости диска Секки.

С другой стороны, показатель ослабления является суммой показателя поглощения и показателя рассеяния излучения в воде:

$$
\varepsilon=k_{a}+\sigma,
$$

где $k_{a}-$ показатель поглощения, $\sigma-$ показатель рассеяния.

Известно [14], что изменчивость показателя поглощения морской воды вблизи длины волны $\lambda=540 \mathrm{~nm}$ относительно мала, и вариации показателя ослабления определяются главным образом вариациями показателя рассеяния $\sigma$. Следовательно, в данной области спектра наблюдается высокая степень корреляции между показателем ослабления $\varepsilon$ и показателем рассеяния $\sigma$, которая описывается выражением

$$
\sigma=0.994 \varepsilon-0.048
$$

Кроме этого, важной ПГХ является индикатриса рассеяния $x(\gamma)$, которая определяется отношением интенсивности излучения, рассеянного элементарным объемом в направлении, составляющем угол $\gamma$ с направлением падающего излучения, к интенсивности излучения, рассеянного тем же объемом равномерно во все стороны. Аппроксимация передней части индикатрисы рассеяния не зависит от типа акватории и описывается уравнением

$$
x(\gamma)=\frac{2}{\mu^{2}} \exp \left[-\frac{\gamma}{\mu}\right] .
$$

Параметр $\mu$ в выражении (7) характеризует вытянутость индикатрисы рассеяния. Чем больше вытянутость индикатрисы рассеяния вперед, тем меньше значение $\mu$.

В морской воде существенная доля потока излучения рассеивается в заднюю полусферу относительно направления распространения. Обратное рассеяние оценивается показателем рассеяния $\sigma_{b}$ в заднюю полусферу или долей излучения, рассеянного в заднюю полусферу, которая определяется как

$$
\varphi_{0}=\frac{\sigma_{b}}{\sigma}
$$

Обратное рассеяние существенно зависит от типа акватории. Однако для акваторий одного типа существует устойчивая зависимость между показателем рассеяния в заднюю полусферу $\sigma_{b}$ и показателями $\sigma$ и $\varepsilon$. В соответствии с моделью О.В. Копелевича в области спектра около $540 \mathrm{~nm}$ справедливы следующие соотношения: для акваторий типа Case 1

$$
\sigma_{b}=0.0183 \varepsilon-0.0094 \sigma
$$

для акваторий типа Case 2

$$
\sigma_{b}=0.018 \varepsilon
$$

Форму индикатрисы рассеяния оценивают значением среднего квадрата угла рассеяния $\left\langle\gamma^{2}\right\rangle$. Независимо от типа акватории в области спектра около $540 \mathrm{~nm}$ этот параметр определяется формулой

$$
\left\langle\gamma^{2}\right\rangle=0.021+0.765 \varphi_{0} .
$$

В этой же области спектра значение параметра, характеризующего вытянутость индикатрисы рассеяния, можно определить выражением

$$
\mu=\sqrt{\frac{\left\langle\gamma^{2}\right\rangle}{6}} .
$$

Таким образом, для каждого типа акваторий получены формулы, позволяющие вычислить любую гидрооптическую характеристику морской воды, зная только глубину $Z_{D}$ видимости диска Секки.

\section{Модель распространения излучения}

Математическая модель представляет собой решение уравнения переноса оптического излучения в малоугловом приближении с учетом принятого модельного описания ПГХ.

Для описания процесса распространения излучения в морской воде справедливо уравнение переноса оптического излучения в рассеивающих средах, которое имеет вид [15]:

$$
\mathbf{n} \nabla L(\mathbf{r}, \mathbf{n})=-\varepsilon L(\mathbf{r}, \mathbf{n})+\frac{\sigma}{4 \pi} \int_{4 \pi} L(\mathbf{r}, \mathbf{m}) x(\widehat{\mathbf{n}, \mathbf{m}}) d \Omega_{m},
$$

где $\mathbf{n} \nabla=n_{x} \frac{\partial}{\partial x}+n_{y} \frac{\partial}{\partial y}+n_{z} \frac{\partial}{\partial z}-$ оператор дифференцирования вдоль луча; $\mathbf{r}, \mathbf{n}$ - соответственно радиус-вектор и вектор направления; $L(\mathbf{r}, \mathbf{n})$ - яркость излучения; $\varepsilon$ - показатель ослабления излучения; $\sigma$ - показатель рассеяния; $x(\widehat{\mathbf{n , m}})-$ индикатриса рассеяния.

Уравнение переноса (13) описывает изменение яркости вдоль луча, распространяющегося в направлении вектора $\mathbf{n}$. Левая часть уравнения характеризует изменение яркости в направлении $\mathbf{n}$, первый член правой части уравнения описывает убывание энергии в результате рассеяния и поглощения, а второй член - прирост энергии в направлении $\mathbf{n}$ из-за рассеяния с направлений $\mathbf{m}$.

Лазерные излучатели, которые используются в составе СТ3, имеют расходимость излучения $2 \alpha_{0}$ не более $2^{\circ}$. Кроме того, в морской среде индикатриса рассеяния существенно вытянута в направлении распространения излучения. Это позволяет применить так называемое 
малоугловое приближение, описывающее поле излучения, которое формируется в рассеивающей среде при прохождении через нее узкого светового пучка.

В малоугловом приближении оператор дифференцирования вдоль луча можно представить как

$$
\mathbf{n} \nabla=\frac{\partial}{\partial z}+\mathbf{n}_{\perp} \nabla_{\perp} ; \quad \mathbf{n}_{\perp} \nabla_{\perp}=n_{x} \frac{\partial}{\partial x}+n_{y} \frac{\partial}{\partial y},
$$

а элемент телесного угла $d \Omega_{m}$ в формуле (13) заменить величиной $d^{2} m_{\perp}$.

Тогда уравнение преобразуется к виду

$$
\begin{aligned}
& \left(\frac{\partial}{\partial z}+\mathbf{n}_{\perp} \nabla_{\perp}\right) L\left(\mathbf{r}_{\perp}, z, \mathbf{n}_{\perp}\right)=-L\left(\mathbf{r}_{\perp}, z, \mathbf{n}_{\perp}\right) \\
& +\frac{\sigma}{4 \pi} \int_{-\infty}^{\infty} L\left(\mathbf{r}_{\perp}, z, \mathbf{n}_{\perp}\right) x\left(\mathbf{n}_{\perp}-\mathbf{m}_{\perp}\right) d^{2} m_{\perp},
\end{aligned}
$$

где в правой части присутствует интеграл свертки.

В связи с этим для решения уравнения (15) целесообразно применить преобразование Фурье. С учетом решения уравнения (15) распределение облученности в плоскости, перпендикулярной оси пучка и находящейся на расстоянии $z$, описывается выражением [16]

$$
\begin{aligned}
& E\left(\mathbf{r}_{\perp} ; z\right)=\frac{1}{2 \pi} \int_{-\infty}^{\infty} \int_{0}(\boldsymbol{v} ; z) \exp \left(-\varepsilon z+\frac{\sigma}{2} \int_{0}^{z} x(v \xi) d \xi\right) \\
& \times \exp \left(i v \mathbf{r}_{\perp}\right) d^{2} v
\end{aligned}
$$

где $\mathbf{r}=\left(\begin{array}{l}x \\ y\end{array}\right)$ - вектор координат, $\boldsymbol{v}=\left(\begin{array}{l}v_{x} \\ v_{y}\end{array}\right)$ - вектор пространственных частот, а расстояние $z$ - параметр.

В декартовой системе координат излучение от источника распространяется в направлении $z$, а плоскость ХOY перпендикулярна направлению распространения излучения. Выражение (16) в декартовой системе координат можно представить в виде

$$
\begin{aligned}
& E(x, y, z)=\int_{-\infty}^{\infty} \widetilde{L}_{0}\left(v_{x}, v_{y} ; z\right) \\
& \times \exp \left(-\varepsilon z+\frac{\sigma}{2} \int_{0}^{z} \tilde{x}\left(\xi \sqrt{v_{x}^{2}+v_{y}^{2}}\right) d \xi\right) \\
& \times \exp \left(i 2 \pi\left(v_{x} x+v_{y} y\right)\right) d v_{x} v_{y},
\end{aligned}
$$

где $\tilde{x}(\rho)=\int_{0}^{\infty} \gamma x(\gamma) J_{0}(\rho \gamma) d \gamma$ - преобразование Ганкеля от индикатрисы рассеяния, причем $\rho=\xi|\nu|$ и $v=\sqrt{v_{x}^{2}+v_{y}^{2}} ; J_{o}(\rho \gamma)-$ функция Бесселя первого рода нулевого порядка; $L_{0}\left(v_{x}, v_{y} ; z\right)$ - пространственночастотный спектр (ПЧС) распределения яркости, создаваемой лазерным источником излучения на расстоянии $z$.
Лазерные источники мощностью $P_{0}$, создающие гауссов пучок, описываются следующим распределением светимости $M(x, y ; z)$ в плоскости $X O Y$ на расстоянии $z$ от источника:

$$
M_{0}(x, y, z)=\frac{P_{0}}{2 \pi z^{2} \alpha_{0}^{2}} \exp \left(-\frac{x^{2}+y^{2}}{2 z^{2} \alpha_{0}^{2}}\right),
$$

где $\alpha_{0}-$ угол расходимости лазерного источника излучения. Если учесть, что телесный угол $\Omega=\frac{\pi\left(x^{2}+y^{2}\right)}{z^{2}}$, то яркость, создаваемую источником, можно определить путем дифференцирования (18):

$$
L_{0}(x, y, z)=\frac{d M_{0}(\Omega ; z)}{d \Omega}=\frac{P_{0}}{4 \pi z^{2} \alpha_{0}^{4}} \exp \left(-\frac{x^{2}+y^{2}}{2 z^{2} \alpha_{0}^{2}}\right),
$$

Вычислив преобразование Фурье от (19), получим выражение для ПЧС распределения яркости в виде

$$
L_{0}(x, y, z)=\frac{P_{0}}{2 \pi \alpha_{l}^{2}} \exp \left[-\pi^{2} z^{2} \alpha_{l}^{2}\left(v_{x}^{2}+v_{y}^{2}\right)\right]
$$

где $\alpha_{l}=\sqrt{2} \alpha_{0}$ - эффективный угол расходимости источника.

Преобразование Ганкеля от индикатрисы рассеяния в переднюю полусферу, которая описывается выражением (7), представляется как

$$
\tilde{x}(\rho)=\int_{0}^{\infty} \gamma \frac{2}{\mu} \exp \left(-\frac{\gamma}{\mu}\right) J_{o}(\rho \gamma) d \gamma=\frac{2}{\left(1+\mu^{2} \rho^{2}\right)^{3 / 2}} .
$$

Для получения аналитического решения уравнения (17) выражение (21) было представлено разложением в ряд Маклорена

$$
\widetilde{x}(\rho)=\frac{2}{\left(1+\mu^{2} \rho^{2}\right)^{3 / 2}}=2-3 \mu^{2} \rho^{2}+\frac{15 \mu^{4} \rho^{4}}{4}+\ldots
$$

С учетом (22) значение интеграла в выражении (17) вычисляется аналитически и равно

$$
\begin{gathered}
\int_{0}^{z} \tilde{x}(\xi v) d \xi=\int_{0}^{z}\left[2-3 \mu^{2} \xi^{2} v^{2}+\frac{15 \mu^{4} \xi^{4} v^{4}}{4}\right] d \xi \\
=2 z-v^{2} z^{3} \mu^{2}+\frac{3}{4} v^{4} z^{5} \mu^{4} .
\end{gathered}
$$

Тогда с учетом формул (20) и (23) результат вычисления обратного преобразования Фурье, описываемого выражением (17), имеет вид

$$
E(r, z)=\frac{P_{0} \exp \left(-k_{a} z\right)}{2 \pi \alpha_{l}^{2} \sqrt{z^{2} \alpha_{l}^{2}+\frac{z^{3} \mu^{2} \sigma}{2 \pi}}} \exp \left(-\frac{2 \pi r^{2}}{z^{2}\left(2 \pi \alpha_{l}^{2}+z \mu^{2} \sigma\right)}\right),
$$

где $r=\sqrt{x^{2}+y^{2}}$.

В формуле (24) множитель $\exp \left(-k_{a} z\right)$ представляет собой коэффициент поглощения, определяющий потери 


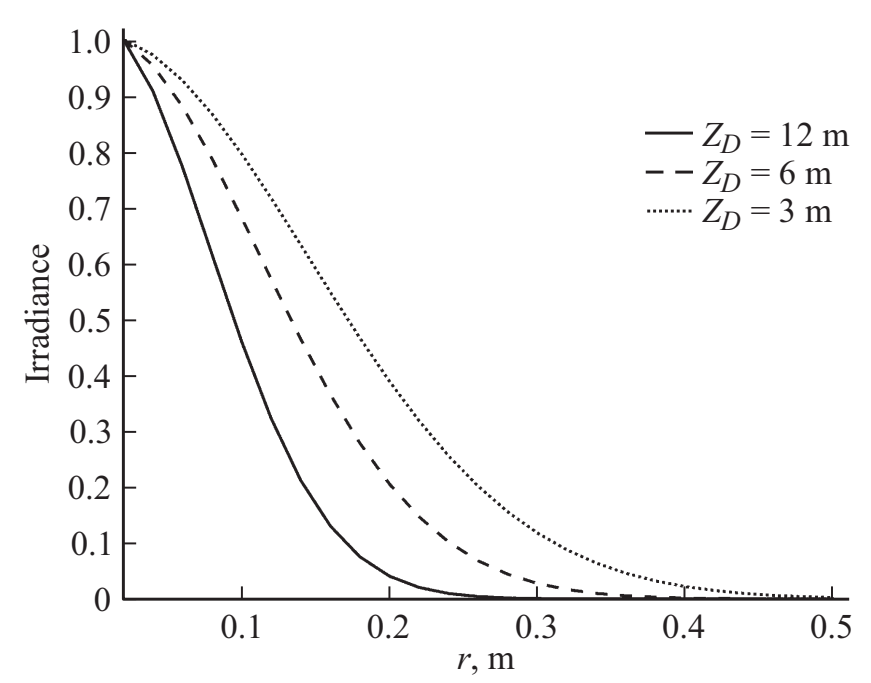

Рис. 2. Графики зависимости распределения нормированной облученности $E_{n}(r)$ на расстоянии $z=20 \mathrm{~m}$ для трех уровней прозрачности воды, характеризуемых параметром $Z_{D}$.

излучения за счет поглощения в слое воды. Коэффициент $z^{2}\left(2 \pi \alpha_{l}^{2}+z \mu^{2} \sigma\right)$ определяет уширение лазерного пучка вследствие рассеяния излучения в слое воды.

Таким образом, зная глубину $Z_{D}$ видимости диска Секки для заданной акватории, по формулам (3)-(12) можно определить гидрооптические характеристики морской воды и по формуле (24) рассчитать распределение облученности для заданной дальности до плоскости предметов.

В качестве иллюстрации на рис. 2 приведены графики распределения нормированной облученности в плоскости, удаленной от источника лазерного излучения на 20 m, для трех уровней прозрачности воды - „прозрачной““, „средней“ и „мутной“, что соответствует глубинам видимости диска Секки 12, 6 и $3 \mathrm{~m}$ соответственно. Расчеты выполнены для акваторий типа Case 2.

\section{Заключение}

В работе представлена математическая модель распространения лазерного излучения в слое морской воды, которая может быть использована при разработке СТЗ активного типа для работы в подводных условиях. Достоинством предложенной модели является относительная простота, так как для ее использования достаточно знать всего один параметр, характеризующий морскую воду для заданной акватории - глубину видимости диска Секки. Для этого определена связь между первичными гидрооптическими характеристиками и глубиной видимости диска Секки, а также выведены аналитические зависимости для расчета пространственного распределения облученности в морской воде на заданном расстоянии от источника лазерного излучения.

\section{Конфликт интересов}

Авторы заявляют, что у них нет конфликта интересов.

\section{Список литературы}

[1] Prieur L., Sathyendranath S. // Limnology and Oceanography. 1981. V. 26. N 4. P. 671-689.

[2] Kopelevich O.V. // Proc. I International Conference „Current Problems in Optics of Natural Waters“ (ONW'2001). / Ed. by Levin I. and Gilbert G. Rozhdestvensky D.S. Optical Society. St. Petersburg, 2001. P. 18-23.

[3] Долин Л.С., Савельев В.А. // Изв. РАН. Физика атмосферы и океана. 2000. Т. 36. № 6. С. 794-801.

[4] Долин Л.С. // Изв. РАН. Физика атмосферы и океана. 2002. T. 38. № 2. C. 273-281.

[5] Arnone R.A., Tucker S., Hilder F. // SPIE Proceedings, Ocean Optics VII. 1984. V. 489. P. 195-201.

[6] Левин И., Копелевич О. // Океанология. 2007. № 3. C. 374-379.

[7] Левин И.М. // Сб. науч. трудов „Фундаментальная и прикладная гидрофизика“. 2007. С. 20-22.

[8] Мухина E.E. Автореф. канд. дисс. М.: МГТУ им. Н.Э. Баумана, 2005. 163 л.

[9] Gould R.W., Arnone R.A., Martinolich P.M. // Appl. Opt. 1999. V. 38. N 12. P. 2377-2383.

[10] Zimin A.V., Rodionov A.A., Rodionov M.A., Pokrovskaya N.E., Frantsuzov O.N. // Proc. V International Conference „Current Problems in Optics of Natural Waters (ONW'2009)“. P. $171-172$.

[11] Власенков Р.Е., Макштас А.П. // Океанография и морской лед. M.: Paulsen, 2011. С. 208-226.

[12] Лубков А.С., Воскресенская Е.Н., Кукушкин А.С. // Материалы научно-практической молодежной конференции „Экологические проблемы Азово-Черноморского региона и комплексное управление прибрежной зоной“. Севастополь, 2014. С. 67-68.

[13] Родионов М.А. Автореф. канд. дисс. СПб., 2012.

[14] Левин И.М. // Фундаментальная и прикладная гидрофизика. 2014. № 3. С. 3-22.

[15] Карасик В.Е., Орлов В.М. Локационные лазерные системы видения. М.: МГТУ им. Н.Э. Баумана, 2013. 479 с.

[16] Карасик В.Е., Мухина Е.Е., Орлов В.М. // Вестник МГТУ им. Н.Э. Баумана. Сер. Приборостроение. 2004. № 1. C. 19-26. 